\title{
RECURRENT ARTERIAL AND VENOUS THROMBOTIC EVENTS IN A PATIENT WITH PSORIASIS. IMPACT OF PAI-1 POLYMORPHISM: A CASE REPORT
}

\author{
Borislav T. Dimitrov, \\ Veronika H. Gincheva ${ }^{1}$, \\ Iva G. Simeonova ${ }^{2}$, \\ Anika I. Ivanova ${ }^{2}$, \\ Maria P. Petkova ${ }^{2}$, \\ Dimitar K. Gospodinov', \\ Regina Komsa-Penkova
}

\author{
Department of Chemistry, \\ Biochemistry, Physics and Biophysics, \\ Medical University - Pleven, \\ Bulgaria \\ ${ }^{1}$ Department of Dermatology, \\ Venereology and Allergology, \\ Medical University - Pleven, \\ Bulgaria \\ ${ }^{2}$ Student of Medicine, \\ Medical University - Pleven, \\ Bulgaria
}

\section{Summary}

Psoriasis is a chronic autoimmune multisystem disease, mainly affecting the skin and joints. Its origin is related to both environmental and genetic factors. The condition affects $1-3 \%$ of the population worldwide. Psoriasis is also associated with cardiovascular risk factors, atherothrombotic events, and markers of hypercoagulation (platelet activation and hyperhomocysteinemia). Venous thromboembolism (VTE) is a widespread severe disease. Both VTE and psoriasis are connected with risk factors for cardiovascular disorders (obesity and hypertension). The incidence of VTE events in patients with psoriasis is higher. Patients with psoriasis should be checked for risk factors (metabolic disorders and cardiovascular diseases).We report a case of a 53-year old man, diagnosed with plaque psoriasis 20 years ago, and a fiveyear history of hypertension. In 2006, he had a stroke, and in 2011 - a heart attack. In 2013 he was diagnosed with thrombophlebitis. The patient was recently diagnosed with Type II diabetes, dyslipidemia and metabolic syndrome. The DNA analysis revealed that the patient was a homozygous carrier of 4G/4G (rs1799889) polymorphism in plasminogen activator inhibitor 1 (PAI1) - a risk factor for thrombophilia. This case is important because of the major comorbidities, more particularly thrombotic events in combination with a prothrombotic mutation.

Key words: psoriasis, venous thromboembolism, PAI-1, comorbidities, thrombosis

\section{Introduction}

Psoriasis is a chronic autoimmune disease with multisystemic manifestations, involving mainly the skin and joints. Its complex origin is associated with environmental and genetic factors. The condition affects $1-3 \%$ of the world's population [1]. Psoriasis provokes epidermal hyperproliferation, abnormal keratinocyte differentiation, angiogenesis with inflammation and dilatation of blood vessels due to excess of T-helper cells - Th1 and Th17. It is related to atherothrombotic events, cardiovascular risk factors and increased coagulability markers like activation of platelets and increased homocysteine [2]. Psoriasis has a major influence on the kidneys, lungs and the cardiovascular system. It can be 
involved in pathological conditions like the metabolic syndrome, and even malignancy.

Venous thromboembolism is a frequent and severe problem, provoked by different clinical conditions (cancer, long-term immobilization, and trauma) [3]. The risk for thromboembolism increases through a variety of mechanisms like inflammation and behavioral risk factors in patients with psoriasis [4, 5]. VTE itself contributes to the increased cardiovascular and total death rate detected in subjects with severe psoriasis [6]. The incidence of VTE events in psoriatic patients is higher as compared to control groups. Only recently, the influence of psoriasis on the risk of VTE has been investigated in detail [7]. A connection between psoriasis and an increased frequency of hyperlipidemia has also been found [8].

Nowadays, the link between metabolic disorders and psoriasis is obvious and physicians have to check out patients with psoriasis, particularly those with severe forms, for metabolic disorders and cardiovascular problems, and administer adequate treatment.

\section{Case Presentation}

We studied a 53-year old white man, with a 20year history of plaque psoriasis, receiving topical treatment with emollient agents and statins. The patient was diagnosed with hypertension $(180 / 110 \mathrm{mmHg})$ five years ago. In 2006, he had a stroke and was treated with Vinpocetine. In 2011, the patient had a heart attack and was treated with isosorbide dinitrate. Two years ago, he was diagnosed with thrombophlebitis on a popliteal vein and was on long-term anticoagulation therapy with warfarin for nearly six months. Recently, he was diagnosed with coxarthrosis and cervical discopathy.

Physical examination revealed well-formed psoriatic plaque lesions on the forearms (Figure 1), feet, elbows, back (Figure 2) and hips (Figure

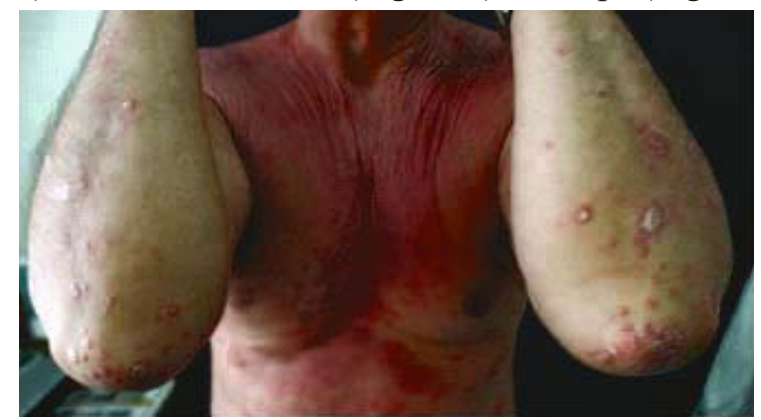

Figure 1. Well-formed psoriatic plaque lesions on the forearms
3). The patient appeared to have abdominal (central) obesity. He is $1.82 \mathrm{~m}$ tall and weighs 115 $\mathrm{kg}$, with waist circumference of $122 \mathrm{~cm}$, hip circumference $-110 \mathrm{~cm}$, and BMI $-34.7 \mathrm{~kg} / \mathrm{m}^{2}$.

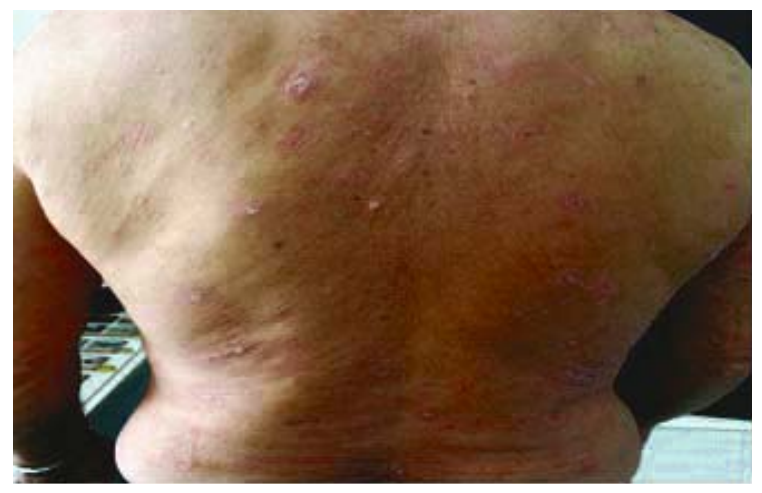

Figure 2. Erythema-papule-squamous plaques with size about $1.5 \mathrm{~cm}$ in diameter, spread on the back, with an intensive accumulation of thick connected squamae

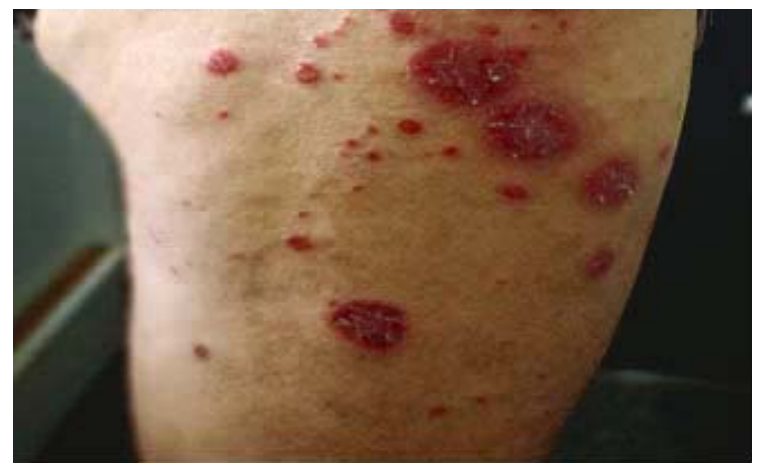

Figure 3. Erythema-papule-squamous plaques with intensive erythema, thick infiltration and accumulation of squamae on the hips

Among the risk factors were moderate regular alcohol consumption, tobacco smoking ten to twenty cigarettes daily for the past 30 years, family-related emotional stress, history of hypertension, a recently diagnosed Type II Diabetes. The family history of the patient included renal disease (father) and cancer (mother). Blood test results are shown in Table 1.

Blood sampling and DNA analysis were carried out as described [9].

Regarding the risk of thrombotic events, the following thrombophilic mutations were investigated: factor V Leiden (FV 1691G $>A$ ), rs6025, factor II prothrombin (FII 20210G $>A$ ), rs1799963, plasminogen activator inhibitor 1 (PAI-1) - 675 4G/5G, rs1799889(-) single nucleotide deletion (SND) in the promotor of PAI-1 gene, polymorphism P1A1/P1A2 in platelet glycoprotein IIb/IIIa, integrin B3 (ITGB3) 
Table 1. Laboratory investigations

\begin{tabular}{|c|c|c|c|c|c|}
\hline Parameters & $\mathrm{V}$ alues & U nits & Min & Max & Interpretation \\
\hline Glucose & 7.53 & $\mathrm{mmol} / 1$ & 4.1 & 6.1 & $\mathrm{H}^{*}$ \\
\hline Urea & 7.66 & $\mathrm{mmol} / \mathrm{l}$ & 0 & 8.1 & - \\
\hline Uric acid & 375 & $\mu \mathrm{mol} / 1$ & 202 & 416 & - \\
\hline Creatinine & 106 & $\mu \mathrm{mol} / 1$ & 80 & 115 & - \\
\hline ASAT & 23.8 & $\mathrm{U} / \mathrm{I}$ & 0 & 40 & - \\
\hline ALAT & 23.5 & $\mathrm{U} / \mathrm{I}$ & 0 & 40 & - \\
\hline GGT & 123.8 & $\mathrm{U} / \mathrm{I}$ & 0 & 50 & $\mathrm{H}$ \\
\hline Cholesterol & 5.99 & $\mathrm{mmol} / \mathrm{l}$ & 0 & 5.2 & $\mathrm{H}$ \\
\hline Triglycerides & 3.3 & $\mathrm{mmol} / \mathrm{l}$ & 0 & 2.3 & $\mathrm{H}$ \\
\hline HDL - cholesterol & 1.32 & $\mathrm{mmol} / \mathrm{l}$ & $>1.7$ & & - \\
\hline Leucocytes & 9.6 & $10^{9} / \mathrm{L}$ & 4.4 & 5.9 & - \\
\hline Erythrocytes & 5.63 & $10^{12} / \mathrm{L}$ & 80 & 115 & - \\
\hline Hemoglobin & 181.0 & $\mathrm{~g} / 1$ & 140 & 180 & $\mathrm{H}$ \\
\hline Hematocrit & 0.526 & $1 / 1$ & 0.40 & 0.53 & - \\
\hline $\mathrm{MCV}$ & 93.0 & $\mathrm{Fi}$ & 82 & 96 & - \\
\hline $\mathrm{MCH}$ & 32.2 & $\mathrm{Pg}$ & 27 & 33 & - \\
\hline Platelets & 170.0 & $10^{9} / \mathrm{L}$ & 150 & 360 & - \\
\hline
\end{tabular}

*H- high

1565T $>$ C, rs5918, methylenetetrahydrofolate reductase (MTHFR) $677 \mathrm{C}>\mathrm{T}$ polymorphism rs1801133 [a single nucleotide polymorphism (SNP) in MTHFR gene].

Treatment and disease progress were as follows: the patient had had elevated blood pressure values $(220 / 140 \mathrm{mmHg})$ since 1997 . In May 2006, he was diagnosed with unstable angina and acute coronary syndrome.

In May 2011, the patient was admitted to hospital and diagnosed with acute myocardial infarction without ST elevation. Selective coronary angiography revealed coronary artery disease and distal left anterior descending artery thrombotic occlusion. Unsuccessful recanalization was performed.

In October of 2006, the patient was diagnosed and treated for cerebral infarction. Computer tomography of the brain and Doppler sonography did not visualize any pathological changes. The patient was treated with losartan potassium, isosorbide dinitrate, simvastatin and acetylsalicylic acid.

Dermatological history and evaluation revealed pathological skin changes in the scalp, back, upper and lower limbs; erythematoussquamous plaques with moderate infiltration, severe desquamation and intense erythema with itching.

The patient had had plaque psoriasis for more than 20 years, and treatment had not been effective. During a hospitalization in 2015 it was found that the psoriatic plaques had covered 30\% of the body surface.

During a recent admission, the patient was treated with Chloropyramine, Eudermol 10, Dithranoli 5\% and unguentum acidi salicylici 5\% for topical application. Psorilys, terbinafiin and zalain were prescribed for treatment after discharge from hospital. For non-drug treatment, ultra violet brulure phototherapy was applied. The patient was discharged with an improvement. 
PCR analysis of prothrombotic and hypofibrinolytic factors (Table 2) showed that the patient is a homozygous carrier of mutant deletion of rs1799889 polymorphism in PAI-1 $4 \mathrm{G} / 5 \mathrm{G}$. This gene is responsible for a mild prothrombotic state.

Table 2. DNA analysis of thrombophilic mutations

\begin{tabular}{lll}
\hline Investigated polymorphism & $\begin{array}{l}\text { Homozygous } \\
\text { Wild }\end{array}$ & $\begin{array}{l}\text { Homozygous } \\
\text { Mutant }\end{array}$ \\
\hline FV $1691 \mathrm{G}>\mathrm{A}$ & $\mathrm{X}$ & \\
\hline FII $20210 \mathrm{G}>\mathrm{A}$ & $\mathrm{X}$ & $\mathrm{X}$ \\
\hline PAI -1 - 675 4G $/ 5 \mathrm{G}$ & & \\
\hline M THFR $677 \mathrm{C}>\mathrm{T}$ & $\mathrm{X}$ & \\
\hline Glycoprotein IIb/IIIa, $1565 \mathrm{~T}>\mathrm{C}$ & $\mathrm{X}$ & \\
\hline
\end{tabular}

\section{Discussion}

A study from 2012 showed that psoriasis increases thrombotic risk events by $40 \%$ in nearly 40000 older females [10]. This study confirms that psoriasis disease is associated with VTE.

Many factors could be linked with thrombotic VTE episodes in psoriatic events. The mechanism that has been recognized to link them to psoriasis combines several factors, including an inflammatory component with the expression of proinflammatory factors/proteins by epigenetic mechanisms. Elevated levels of mediators of inflammation like C-reactive protein, ceruloplasmin, cytokines and eosinophilia in psoriatic patients could stimulate a hypercoagulable state $[11,12]$.

PAI-1 is another important molecule, released by endothelial cells. It is also stored and released from platelets upon activation, which could worsen the symptoms [13]. This coagulation factor downregulates fibrinolysis through inhibiting plasminogen activation and its transformation to plasmin by urokinase and tissue-plasminogen activator [14]. PAI-1 is a member of the group of serine protease inhibitors (SERPINE1). There are several PAI-1 polymorphisms of clinical significance described so far. The most common is the rs1799889 PAI-1 gene polymorphism (insertion/deletion at -675 position in the promoter region), which was found to contribute to prothrombotic risk and heart attack [15].

PAI-1 is linked with vascular inflammation and atherothrombosis [16]. There is data proving the relationship between its elevated levels and metabolic syndrome. Increased PAI-1 activity is observed in atherosclerosis, especially in patients with obesity or diabetes mellitus type II [17]. Elevated PAI-1 levels are related to high weight, as PAI- 1 is produced by fat depots [18].

The patient we investigated is a homozygous carrier of $4 \mathrm{G}$ polymorphism, a smoker, with obesity, diabetes and dyslipidemia - all being risk factors that increase the risk for arterial and venous thrombosis.

There is a variety of factors that can increase the risk for thrombosis in psoriatic patients. Among them are factors like age, improper response to therapy and severity of the disease. There is evidence of statistically significant increased risk for VTE and cardiovascular disease (CVD) in patients with psoriasis $[19,20]$. The increase in homocysteine, due to the condition or related to its treatment provokes vascular and endothelial damage and changes in the coagulation profile by inhibiting thrombomodulin, protein $\mathrm{C}$ anticoagulant pathway, receptors for tissue plasminogen activator, thus inducing thrombosis [21]. Carriage of MTHFR polymorphism contributes to higher homocysteine levels, and these patients need folic acid supplementation [22]. Fortunately, our patient is not a carrier of this polymorphism.

Statins used as a medication for psoriasis have an anti-inflammatory effect. They have a positive effect on psoriatic skin lesions and may also decrease VTE risk, particularly in atherosclerotic patients [23]. 
Because of the risk for thrombosis in patients with psoriasis, administration of anticoagulation therapy is not fully justified. Furthermore, patients with psoriasis may already be on cardiopreventive therapy, such as statins and weight loss, which may also lower the risk of VTE [10].

It is also worth mentioning that anti-TNF-a agents like antibodies, used to treat psoriasis, may have a cardio-protective effect [24, 25].

There is a high probability that the recurrent thrombotic events have been provoked by psoriasis in this case. Due to the obvious risk factor (high cholesterol, high triacylglycerols, diabetes and 4G/4G PAI-1 polymorphism and psoriasis), this patient should be under observation for future thrombotoc events. Moreover, comorbidities may further worsen his health.

This case is important because of the presence of major comorbidities and thrombotic events in particular. The question is what the correlation between the accompanying diseases and psoriasis itself is, and whether the recurrent thrombotic events are of multifactorial origin. Could the treatment for psoriasis provoke thrombotic events?

\section{Conclusions}

In conclusion, this case summarizes a relation between continuous inflammation and increased risk of VTE, proposing that patients with psoriasis might be at a higher risk for VTE incidents. This pathological condition is not completely understood, but the possibility of thrombosis in psoriatic patients should be taken into account. There is evidence of the link between psoriasis and disorders of metabolism, which has changed treatment given to psoriatic patients. Physicians should examine patients with psoriasis for metabolic disorders, cardiovascular and thrombotic risk factors before administering treatment. Although prophylaxis against low fibrinolysis is not justified, doctors should be prepared for all eventualities. The inheritance for thrombotic risk factors has to be investigated, as it contributes to the multifactorial risk of comorbidities.

\section{References}

1. Azfar R, Gelfand J. Psoriasis and metabolic disease: Epidemiology and pathophysiology. Curr Opin Rheumatol. 2008;20(4):416-22.

2. Friedewald E, Cather C, Gelfand M, Gordon B, Gibbons H, Grundy S. AJC editor's consensus: psoriasis and coronary artery disease. Am J Cardiol. 2008;102(12):1631-43.

3. Goldhaber S. Risk factors for venous thromboembolism. J Am Coll Cardiol. 2010;56(1):1-7.

4. Machlus K, Aleman M, Wolberg A. Update on venous thromboembolism: risk factors, mechanisms, and treatments. Arterioscler ThrombbVasc Biol. 2011;31(3):476-8.

5. Holst A, Jensen G, Prescott E. Risk factors for venous thromboembolism: results from the Copenhagen City Heart Study. Circulation. 2010;121:1896-903.

6. Mallbris L, Akre O, Granath F, Yin L, Lindelof B, Eubom A, et al. Increased risk for cardiovascular mortality in psoriasis inpatients but not in outpatients. Eur J Epidemiol. 2004;19(3):225-30.

7. Reitsma P, Versteeg H, Middeldorp S. Mechanistic view of risk factors for venous thromboembolism. Arterioscler Thromb Vasc Biol. 2012;32(3):563-8.

8. KimballA, Gladman D, Gelfand J, Gordon K, Horn E, Korman N, et al. National psoriasis foundation clinical consensus on psoriasis comorbidities and recommendations for screening. J Am Acad Dermatol. 2008;58(6):1031-42.

9. Komsa-Penkova R, Kovacheva-Kotseva K, Angelova S, Savov A, Simeonova M. [Selected methods of DNA analysis and clinical applications]. 1st ed. Pleven: MU-Pleven; 2004. Bulgarian.

10. Lutsey P, Prizment A, Folsom A. Psoriasis is associated with a greater risk of incident venous thromboembolism: the Iowa Women's Health Study. J Tromb Haemost. 2012;10(4):708-11.

11. Karabudak O, Eralp R, Akyol A, Solmazgul E, Dogan B, Harmanyeri Y. Inflammation and hypercoagulable state in adult psoriatic men. Acta Derm Venereol. 2008;88(4):337-40.

12. Ko JH, Lin JW, Hui RC. Acute pulmonary embolism in a patient with hypereosinophilia and psoriasis. Chang Gung Med J. 2011;34(6):17-23.

13. Vaughan D. PAI-1 and atherotrombosis. J Tromb Haemost. 2005;(8):1879-83.

14. Mehta R, Shapiro A. Plasminogen activator inhibitor type 1 deficiency. Haemophilia. 2008;14(6):1255-60.

15. Tsantes A, Nikolopoulos G, Bagos P, Bonovas S, Kopterides P, Vaiopoulos G. The effect of the plasminogen activator inhibitor- $14 \mathrm{G} / 5 \mathrm{G}$ polymorphism on the thrombotic risk. Thromb Res. 2008;122(6):736-42. 
16. Aso Y. Plasminogen activator inhibitor (PAI)-1 in vascular inflammation and thrombosis. Front Biosci. 2007;12(8):2957-66.

17. Juhan-Vague I, Alessi MC, Mavri A, Morange P. Plasminogen activator inhibitor-1, inflammation, obesity, insulin resistance and vascular risk. J Thromb Haemost. 2003;1(7):1575-9.

18. Alessi MC, Poggi M, Juhan-Vague I. Plasminogen activator inhibitor-1, adipose tissue and insulin resistance. Curr Opin Lipidol. 2007;18(3):240-5.

19. Ungprasert P, Sanguankeo A, Upala S, Suksaranjit P. Psoriasis and risk of venous thromboembolism: a systematic review and meta-analysis. QJM. 2014;107(10):793-7.

20. Catherine N, Chiu M. Psoriasis and comorbidities: links and risks. Clin Cosmet Investig Dermatol. 2014;7:119-32.

21. Harpel P, Zhang X, Borth W. Homocysteine and hemostasis: pathogenetic mechanisms predisposing to thrombosis. J Nutr. 1996;126(4 Suppl):1285S-9S.

22. Casas J, Bautista L, Smeeth L, Sharma P, Hingorani A. Homocysteine and stroke: evidence on a causal link from mendelian randomisation. Lancet. 2005;365(9455):224-32.

23. Wakkee M, Thio H, Prens E, Sijbrands E, Neumann H. Unfavorable cardiovascular risk profiles in untreated and treated psoriasis patients. Atherosclerosis. 2007;190(1):1-9.

24. Oh C, Das K, Gottlieb A. Treatment with antitumor necrosis factor $\alpha(\mathrm{TNF}-\alpha)$ monoclonal antibody dramatically decreases the clinical activity of psoriasis lesions. J Am Acad Dermatol. 2000;42(5):829-30.

25. Ryan C, Menter A. Psoriasis and cardiovascular disorders. G Ital Dermatol Venereol. 2012;147:179-87. 